\title{
https://doi.org/10.46813/2021-131-036 \\ ION-CYCLOTRON ABSORPTION OF FAST WAVES IN A CYLINDRICAL CURRENT-CARRYING PLASMA
}

\author{
N.I. Grishanov ${ }^{1,2}$, N.A. Azarenkov ${ }^{1}$ \\ ${ }^{1}$ V.N. Karazin Kharkiv National University, Kharkiv, Ukraine; \\ ${ }^{2}$ Ukrainian State University of Railway Transport, Kharkiv, Ukraine
}

Influence of a longitudinal stationary current on the absorption and the radial structure of fast waves in a cylindrical current-carrying plasma is discussed. To evaluate the dispersion equation for fast waves, there was used the dielectric tensor taking into account the radial current structure and geometry of the confining helical magnetic field by the plasma safety factor. It is shown that the damping rate of fast waves in a non-equilibrium current-carrying plasma differ from those for an equilibrium plasma column in a homogeneous magnetic field nearby the cutoffs and resonances due to the rotational transformation (including shear-effects) of the helical magnetic field lines.

PACS: 52.50.Qt

\section{INTRODUCTION}

The fast waves (often referred as the fast magnetosonic or compressional Alfvén waves) are interesting for scientists both academically and in applications for fusion plasmas. They can be used for an additional plasma heating in tokamaks, stellarators and open mirror-traps in the frequency range of ion-cyclotron resonances (ICR) and are believed to be responsible for ion-cyclotron emission there. Presently, the linear theory is very well developed [1] for any plane waves in a uniform magnetized plasma confined by a straight magnetic field. However, the plane waves are not suitable for cylindrical and toroidal plasma models. In this connection, the theory of fast waves was significantly advanced at the end of 20th century using the models of cylindrical and quasi-cylindrical waves in the cylindrical [2-5] and toroidal [5-10] plasma configurations. It was shown that the eigenfrequencies of fast waves are defined by the contribution of bulk particles (mainly ions) to the transverse dielectric tensor components. As some restriction of these investigations is an assumption that the dielectric tensor $\varepsilon_{i k}$ for waves in any cold current-carrying magnetized plasma has the same (invariant) form, i.e., like as for plane waves in a straight magnetic field [1].

However, the current-carrying plasma is not in the stable equilibrium. Therefore, wave analysis in the currentcarrying plasmas should take into account the influence of rotational transformation of the stationary magnetic field lines (including the shear-effects) on the dielectric properties of plasmas in the helical magnetic field. The main goal of our paper is to describe the fast waves in the cylindrical current-carrying plasmas using the approach developed in Refs. [3, 11-14].

\section{PLASMA MODEL}

The simplest model of tokamaks is a magnetized plasma cylinder (radius $a$ ) with identical ends in the helical magnetic field, where the ohmic current $\mathbf{j}_{0}$ generates the poloidal magnetic field $\mathbf{H}_{0 \theta}=H_{0 \theta} \mathbf{e}_{\theta}$ in addition to the longitudinal $\mathbf{H}_{0 z}=H_{0 z} \mathbf{e}_{z}$, where $\mathbf{e}_{r}, \mathbf{e}_{\theta}$, and $\mathbf{e}_{z}$ are the unit vectors along the axis $r, \theta$ and $z$ of the cylindrical

coordinates. In this case, the length of plasma cylinder is equal to $2 \pi R_{0}$, where $R_{0}$ corresponds to a major tokamak radius. As a result, the stationary field $\mathbf{H}_{0}=\mathbf{H}_{0 \theta}+\mathbf{H}_{0 z}$ becomes helical with a rotational transformation, allowing to take into account the sheareffects and the radial profiles of ohmic current by the radial dependence of plasma safety factor $q(r)=r H_{0 z} /\left(R_{0} H_{0 \theta}\right)$ and its derivatives.

Describing such plasma model [11-14] we assume that the steady-state current $\left(\mathbf{j}_{0} \| \mathbf{H}_{\mathrm{O}}\right)$ is created by electrons having the velocity $v_{0 e}=v_{0}$, whereas $v_{0 i}=0$ for heavy ions. In this case, according to Ampere's law,

$$
\left(\nabla \times \mathbf{H}_{0}\right) \cdot \mathbf{h}=\frac{4 \pi}{c} j_{0}=-\frac{4 \pi}{c} N_{0 e} e v_{0},
$$

where

$$
\mathbf{h}=\frac{\mathbf{H}_{0}}{H_{0}}, \quad v_{0}=-\frac{\kappa_{2} H_{0} c}{4 \pi n_{0 e} e} .
$$

Here $n_{0 e}$ is the electron density, $e$ is the elementary charge, and magnetic field parameter $\kappa_{2}$ is equal to

$$
\kappa_{2}=2 \frac{h_{z} h_{\theta}}{r}\left(1-\frac{r}{2 q} \frac{d q}{d r}\right) .
$$

To evaluate the dispersion relations for eigenmodes in the current-carrying plasma, as usual, we should resolve the Maxwell's equations for perturbed electric field $(\mathbf{E})$, magnetic field $(\mathbf{H})$ and current density $(\mathbf{j})$ components. Further, we use the normal $A_{n}$, binormal $A_{b}$ and parallel $A_{h}$ projections relative to $\mathbf{H}_{0}$ for the vector values $\mathbf{A}=\{\mathbf{E}, \mathbf{H}, \mathbf{j}\}=A_{n} \mathbf{n}+A_{b} \mathbf{b}+A_{h} \mathbf{h}$ :

$$
A_{1} \equiv A_{n}=\mathbf{A} \cdot \mathbf{n}, \quad A_{2} \equiv A_{b}=\mathbf{A} \cdot \mathbf{b}, \quad A_{3} \equiv A_{h}=\mathbf{A} \cdot \mathbf{h},
$$

where $\mathbf{n}, \mathbf{b}, \mathbf{h}$ are the normal, binormal and parallel unit vectors relative to $\mathbf{H}_{0}$ :

$$
\begin{aligned}
& \mathbf{h}=\mathbf{H}_{0} / H_{0}=h_{\theta} \mathbf{e}_{\theta}+h_{z} \mathbf{e}_{z}, \\
& \mathbf{n}=\mathbf{b} \times \mathbf{h}=\mathbf{e}_{r}, \\
& \mathbf{b}=\mathbf{h} \times \mathbf{n}=h_{z} \mathbf{e}_{\theta}-h_{\theta} \mathbf{e}_{z},
\end{aligned}
$$

accounting for that $b_{r}=h_{r}=0, b_{\theta}=h_{z}$ and $b_{z}=-h_{\theta}$.

\section{WAVE'S EQUATIONS}

Assuming the smallness of a poloidal magnetic field, $h_{\theta} \ll h_{z}$, the differential Maxwell's equations for short wave perturbations, proportional to 
$\{\mathbf{E}, \mathbf{H}, \mathbf{j}\} \sim \exp \left(-\mathrm{i} \omega t+\mathrm{i} m \theta+\mathrm{i} n z / R_{0}+\mathrm{i} \int k_{r} d r\right)$,

can be reduced, in the geometric optic approximation, to the set of linear algebraic equations

$$
\begin{gathered}
H_{1}=N_{b} E_{3}-N_{\|} E_{2}, \\
H_{2}=N_{\|} E_{1}+i N_{1} E_{2}-N_{r} E_{3}, \\
H_{3}=-N_{b} E_{1}+N_{r} E_{2}-i N_{2} E_{3}, \\
N_{\|} H_{2}-N_{b} H_{3}=\varepsilon_{11} E_{1}+\varepsilon_{12} E_{2}+\varepsilon_{13} E_{3}, \\
-N_{\|} H_{1}-i N_{1} H_{2}+N_{r} H_{3}=\varepsilon_{21} E_{1}+\varepsilon_{22} E_{2}+\varepsilon_{23} E_{3}, \\
N_{b} H_{1}-N_{r} H_{2}+i N_{2} H_{3}=\varepsilon_{31} E_{1}+\varepsilon_{32} E_{2}+\varepsilon_{33} E_{3} .
\end{gathered}
$$

Here $N_{r}=\frac{k_{r} c}{\omega}, \quad N_{b}=\frac{k_{b} c}{\omega}, \quad N_{\|}=\frac{k_{\|} c}{\omega}$ are the radial (normal), binormal and parallel refractive index components, corresponding to the radial $\left(k_{r}\right)$, binormal $\left(k_{b}=\frac{m h_{z}}{r}-\frac{n h_{\theta}}{R_{0}}\right)$ and parallel $\left(k_{\|}=\frac{m h_{\theta}}{r}+\frac{n h_{z}}{R_{0}}\right)$ wavenumbers; $m$ and $n$ are the poloidal and toroidal eigenmode numbers;

$$
N_{1}=\frac{\kappa_{1} c}{\omega}, \quad N_{2}=\frac{\kappa_{2} c}{\omega}, \quad \kappa_{1}=\frac{h_{z} h_{\theta}}{q} \frac{d q}{d r},
$$

where $\kappa_{1}-$ parameter is defined by the shear of magnetic field lines $(\sim d q / d r)$ in the explicit form.

Deriving Eqs. (7) the perturbed current density components were excluded by the connection $4 \pi \mathrm{ij} j_{i} / \omega=\left(\varepsilon_{i k}-\delta_{i k}\right) E_{k}$, where subscribed indexes $i, k=1,2,3$ numerate the $\mathbf{n}, \mathbf{b}, \mathbf{h}$ projections of the vector values, Eqs. (4); $\delta_{i k}$ are the Kronecker symbols.

Contribution of cold particles in the current-carrying plasma to the dielectric tensor $\varepsilon_{i k}$ for waves in the frequency range below the electron-cyclotron frequency $\left(\omega<<\Omega_{e} \mid\right)$ can be expressed as $[11,12]$ :

$$
\begin{gathered}
\varepsilon_{11}=\varepsilon_{\perp}=1+\sum_{\alpha}^{e, i_{1}, i_{2}, \ldots} \frac{\omega_{p \alpha}^{2}}{\Omega_{\alpha}^{2}-\omega^{2}} \simeq 1+\sum_{\beta}^{i_{1}, i_{2}, \ldots} \frac{\omega_{p \beta}^{2}}{\Omega_{\beta}^{2}-\omega^{2}}, \\
\varepsilon_{22}=\varepsilon_{\perp}-N_{1} N_{2}, \\
\varepsilon_{12}=-\varepsilon_{21}=\mathrm{i} \sum_{\alpha}^{e, i_{1}, i_{2}, \ldots} \frac{\omega_{p \alpha}^{2} \Omega_{\alpha}}{\omega\left(\Omega_{\alpha}^{2}-\omega^{2}\right)}-\mathrm{i} N_{\|} N_{2} \simeq \\
\simeq \mathrm{i} \sum_{\beta}^{i_{1}, i_{2}, \ldots} \frac{\omega_{p \beta}^{2} \omega}{\Omega_{\beta}\left(\Omega_{\beta}^{2}-\omega^{2}\right)}-\mathrm{i} N_{\|} N_{2}, \\
\varepsilon_{13}=-\varepsilon_{31}=\mathrm{i} N_{b} N_{2}, \quad \varepsilon_{23}=-\varepsilon_{32}=-\mathrm{i} N_{r} N_{2}, \\
\varepsilon_{33}=\varepsilon_{\| 1}=1-\sum_{\alpha}^{e, i_{1}, i_{2}, \ldots .} \frac{\omega_{p \alpha}^{2}}{\omega^{2}} \simeq 1-\frac{\omega_{p e}^{2}}{\omega^{2}} .
\end{gathered}
$$

Here $\omega_{p \alpha}^{2}=4 \pi N_{0 \alpha} \tilde{q}_{\alpha}^{2} / M_{\alpha}$ is the squared Langmuir frequency of $\alpha$-kind particles, $\Omega_{\alpha}=\tilde{q}_{\alpha} H_{0} / M_{\alpha} c$ is the cyclotron (Larmor) frequency of ions (single or multiple species, $\left.\alpha=i_{1}, i_{2}, \ldots, \Omega_{i}>0\right)$ and electrons ( $\alpha=e$, $\left.\Omega_{e}<0\right) ; M_{\alpha}, N_{0 \alpha}$ and $\tilde{q}_{\alpha}$ are the mass, density and charge of $\alpha$-kind plasma particles; $H_{0}=\sqrt{H_{0 z}^{2}+H_{0 \theta}^{2}}$ is the stationary magnetic field module; $c$ is the speed of light. As we see, the transverse component $\varepsilon_{11} \equiv \varepsilon_{\perp}$ is defined mainly by the contribution of plasma ions; the longitudinal $\varepsilon_{33} \equiv \varepsilon_{\|}$and off-diagonal $\varepsilon_{13}, \varepsilon_{31}, \varepsilon_{23}, \varepsilon_{32}$ components - by the current-carrying electrons; $\varepsilon_{22}$ and gyrotropic components $\varepsilon_{12}$ and $\varepsilon_{21}$ - by both the ions and electrons. Of course, if $\mathbf{j}_{0}=0, h_{\theta}=0, h_{z}=1, \kappa_{1}=0$, $\kappa_{2}=0$, this dielectric tensor can be reduced to $\varepsilon_{i k}$ for waves in a cold equilibrium collisionless plasma in a uniform magnetic field [1].

Excluding the magnetic field components by the Faraday's law [first three equations in (7)], we obtain the following wave equations for $E_{1}, E_{2}$, and $E_{3}$ :

$$
\begin{aligned}
\left(\varepsilon_{\perp}-N_{\|}^{2}-N_{b}^{2}\right) E_{1}+\left(N_{r} N_{b}+\mathrm{i} g\right) E_{2}+N_{r} N_{\|} E_{3}=0, & \\
\left(N_{r} N_{b}-\mathrm{i} g\right) E_{1}+\left(\varepsilon_{\perp}-N_{r}^{2}-\right. & \left.N_{b}^{2}-N_{0} N_{1}\right) E_{2}+ \\
& +\left(N_{b} N_{\|}-i N_{r} N_{1}\right) E_{3}=0,
\end{aligned}
$$

$$
N_{r} N_{\|} E_{1}+\left(N_{b} N_{\|}+\mathrm{i} N_{r} N_{1}\right) E_{2}+\left(\varepsilon_{\|}-N_{r}^{2}-N_{b}^{2}-N_{2}^{2}\right) E_{3}=0,
$$

where the additional designation

$$
g=\sum_{\beta}^{i_{1}, i_{2}, \ldots} \frac{\omega_{p \beta}^{2} \omega}{\Omega_{\beta}\left(\Omega_{\beta}^{2}-\omega^{2}\right)}-N_{\|} N_{0}
$$

is introduced instead of $\varepsilon_{12}-\mathrm{i} N_{\|} N_{1} \equiv \mathrm{i} g$. Note, the new parameter $N_{0} \equiv N_{2}+N_{1}$ is independent from the magnetic shear. Moreover, the term $N_{0} N_{\|}$can be comparable and larger than the ion contribution to $g$ for low frequency waves $\left(\omega<<\Omega_{i}\right)$ far from the rational magnetic surfaces, where $m+n q(r)>>1$.

\section{DISPERSION RELATIONS}

As usual, the squared radial refractive index $N_{r}^{2}$ of eigenmodes in the current-carrying plasma cylinder can be derived from the corresponding biquadratic dispersion equation [i.e., when the determinant of Eqs. (10) is equal to zero]:

$$
A N_{r}^{4}-B N_{r}^{2}+C=0
$$

where

$$
\begin{aligned}
A= & \varepsilon_{\perp}, \\
B= & \left(\varepsilon_{\|}+\varepsilon_{\perp}-N_{2}^{2}\right)\left(\varepsilon_{\perp}-N_{\|}^{2}\right)-g^{2}-N_{1} N_{2}\left(\varepsilon_{\perp}-N_{b}^{2}\right)+ \\
& +N_{\|}^{2} N_{1}^{2}-2 N_{b}^{2} \varepsilon_{\perp}-2 g N_{\|} N_{1}, \\
C= & \left(\varepsilon_{\|}-N_{b}^{2}-N_{2}^{2}\right)\left[\left(\varepsilon_{\perp}-N_{\|}^{2}-N_{b}^{2}\right)\left(\varepsilon_{\perp}-N_{\|}^{2}-N_{0} N_{1}\right)-g^{2}\right]- \\
& -N_{b}^{2} N_{\|}^{2}\left(\varepsilon_{\perp}-N_{\|}^{2}-N_{b}^{2}\right) .
\end{aligned}
$$

This dispersion equation allows us to determine the radial structure of eigenmodes (by $k_{r}$, as a boundary value problem) in dependence on the given $m, n$ and $\omega$ at the considered magnetic surface [by $r=$ const under the given radial profiles of $N_{0 \alpha}(r)$ and $q(r)$ ]. The shear corrections $N_{0} N_{1}$ in $C$ are smaller than $\varepsilon_{\perp}$, but they can be comparable with $N_{\|}^{2}$, e.g., nearby the rational magnetic surfaces, where $m+n q(r) \approx 0$. It should be noted, that assuming $N_{0}=N_{1}=N_{2}=0$ into $B$ and $C$ coefficients, Eq. (12) can be easily reduced to the dispersion equation in Ref. [7] suitable for plane (and cylindrical) slow and fast waves in the equilibrium magnetized plasmas held by a uniform magnetic field, where $h_{\theta}=0, k_{r}=k_{x}, k_{\|}=n / R_{0}=k_{z}, k_{b}=k_{\theta}=m / r=k_{y}$.

Two roots of Eq. (12) are equal to

$$
N_{r \pm}^{2}=\frac{B \pm \sqrt{B^{2}-4 A C}}{2 A},
$$


corresponding [7] to slow waves $\left(N_{r S W}^{2} \equiv N_{r+}^{2}\right)$ and fast waves $\left(N_{r F W}^{2} \equiv N_{r-}^{2}\right.$ ), respectively. Using inequalities $\left|\varepsilon_{\perp}\right|,|g|<<\left|\varepsilon_{\|}\right|$, Eqs. (14) can be simplified to

$$
\begin{aligned}
& N_{r S W}^{2} \simeq N_{b}^{2}+\frac{\varepsilon_{\|}}{\varepsilon_{\perp}}\left(\varepsilon_{\perp}-N_{\|}^{2}\right), \\
& N_{r F W}^{2} \simeq \frac{\left(\varepsilon_{\perp}-N_{\|}^{2}-N_{b}^{2}\right)\left(\varepsilon_{\perp}-N_{\|}^{2}-N_{0} N_{1}\right)-g^{2}}{\varepsilon_{\perp}-N_{\|}^{2}} .
\end{aligned}
$$

\section{ICR ABSORPTION OF FAST WAVES}

As is well known [1], the growth/damping rate of any waves is determined by the anti-hermitic part of dielectric tensor elements in the considered plasma models. Since the dielectric tensor $\varepsilon_{j k}$ in Eqs. (9) is hermitic, the slow and fast waves in our cold currentcarrying plasma cylinder must be pure harmonicperiodic. However, we should remember about the possible two principal collisional and collisionless wave dissipation mechanisms in any plasma model.

The collisional mechanism is connected with resistive (ohmic) conductivity of cold plasma models due to electron-ion friction as a result of effective electron-ion collisions. Resistive absorption both the fast and slow waves in magnetized plasmas usually is small. It can be substantial [7] only for slow waves at the plasma periphery under the conditions where the particle density is quite large and temperature is very law. The detailed analysis of influence of collisional effects on the cyclotron wave-particle interactions in magnetized plasmas of tokamaks and stellarators has been done at Ref. [8].

Other collisionless wave dissipation mechanisms are connected with the resonance wave-particle interactions in the high-temperature plasmas when collisional (resistive) wave damping become ineffective. In this case, the kinetic wave theory should be used to estimate the contribution of resonant particles to the anti-hermitic parts of $\varepsilon_{j k}$. As one can easily verify in section 4 , the collisionless damping rate of slow waves (i.e., imaginary part of radial refractive index, $\left.\operatorname{Im} N_{r S W} \sim \operatorname{Im} \varepsilon_{\|}\right)$in a high-temperature plasma can be defined by the contribution of resonant electrons to the imaginary part (or anti-hermitic part) of the parallel dielectric tensor element $\varepsilon_{\|}$:

$$
\begin{gathered}
\varepsilon_{\|}=1+\sum_{\alpha}^{e, i} \frac{2 \omega_{p \alpha}^{2}}{k_{\|}^{2} v_{T s}^{2}}\left[1+\mathrm{i} \sqrt{\pi} \varsigma_{\alpha} W\left(\varsigma_{\alpha}\right)\right] \approx \\
\approx \frac{2 \omega_{p e}^{2}}{k_{\|}^{2} v_{T e}^{2}}\left[1+\mathrm{i} \sqrt{\pi} \varsigma_{e} W\left(\varsigma_{e}\right)\right] .
\end{gathered}
$$

Here

$$
W(\varsigma)=\mathrm{e}^{-\varsigma^{2}}\left(1+\frac{2 \mathrm{i}}{\sqrt{\pi}} \int_{0}^{\varsigma} e^{t^{2}} d t\right)
$$

is the probability integral for the complex arguments $\zeta$ :

$$
\varsigma_{e}=\frac{\omega-k_{\|} v_{0}}{k_{\|} v_{T e}},
$$

where $[12,13]$ the thermal velocity of hot electrons is defined by their temperature $T_{0 e}, v_{T e}=\sqrt{2 T_{0 e} / M_{e}}$, and the current velocity is determined in Eq. (2). The corresponding resonance conditions for waves with phase velocity $\omega / k_{\|}$and particles having the same parallel velocity $v_{\|}=\omega / k_{\|}$are known as the Cherenkov resonance conditions: $\omega=k_{\|} v_{\|}$. In this case, the fieldaligned electric field component $E_{\|}=\mathbf{h} \cdot \mathbf{E}$ of waves effectively interacts with plasma particles moving along the $\mathbf{H}_{0}$ field lines, and the wave absorption mechanism itself is named as the electron (or ion) Landau damping.

However, as was mentioned above, in a magnetized collisionless plasma there is another wave dissipation mechanism connected with cyclotron wave-particle interactions under the conditions when the transverse electric field components $\left(E_{1}+\mathrm{i} \ell E_{2}\right)$ can effectively interact with plasma particles moving along the magnetic field lines with the parallel velocities $\nu_{\|} \sim\left(\omega-\ell \Omega_{\alpha}\right) / k_{\|}$, where the indices $\alpha=e, \alpha=i$ mark the cyclotron frequencies of electrons and ions, and the integer values $\ell= \pm 1, \pm 2, \ldots$ correspond to the numbers of cyclotron resonances. The corresponding single wave-particle resonance conditions in magnetized plasmas confined by the uniform (straight) magnetic field are well-known: $\omega-k_{\|} v_{\|}=\ell \Omega_{\alpha}$, where $k_{\|} \equiv k_{z}$, since $h_{\theta}=0$. Considering the cylindrical magnetized current-carrying plasmas (in the helical magnetic field) we should take into account that the cyclotron waveparticle resonance conditions there have some another form $[13,14]$ :

$$
\omega-k_{\|} v_{\|}=\ell\left[\Omega_{s}+\kappa v_{\alpha \|}\right] .
$$

A specific feature of current-carrying plasmas is the $\kappa$ shift,

$$
\kappa=2 \frac{h_{\theta} h_{z}}{r}\left(1-\frac{r}{4 q} \frac{d q}{d r}\right),
$$

in the parallel projection of the wave vector, $k_{\|}$, due to the curvature of the external magnetic field lines in the plasma with ohmic current. This $\kappa$-shift is responsible for the difference between the resonance conditions in the plasmas with straight and helical magnetic fields. Evidently, if $\kappa=0$ or $h_{\theta}=0$, i.e., in the absence of ohmic current, the cyclotron resonance conditions automatically reduce to the expression $\omega-k_{\|} \nu_{\|}=\ell \Omega_{\alpha}$, for plasmas in a uniform magnetic field, where $k_{\|} \rightarrow k_{z}$.

In this section we consider the ion-cyclotron resonance absorption of fast waves in a cylindrical current-carrying plasma nearby the first (principal, fundamental, $\ell=1$ ) cyclotron frequency harmonic. Unfortunately, there are no correct expressions of $\varepsilon_{j k}$ elements suitable for waves with $\omega \sim \ell \Omega_{\alpha}$ (under $|\ell| \geq 2$ ) in the current-carrying plasma models. As for case of $|\ell|=1$, the contributions of resonant ions to $\varepsilon_{j k}$ can be derived by solving [14] the Vlasov equation for perturbed distribution functions of plasma particles in a cylindrical current-carrying plasma under the arbitrary values of parameter $k_{\|} v_{\|} /\left(\omega-\Omega_{i}\right)$. In this case, we obtain [13] the following dielectric tensor elements $\varepsilon_{j k}^{(i)}$ : 


$$
\begin{aligned}
& \varepsilon_{11}^{(i)}=\varepsilon_{22}^{(i)}=\frac{\omega_{p i}^{2}}{2 \omega}\left[\frac{\mathrm{i} \sqrt{\pi} W\left(\varsigma_{i}\right)}{\left|k_{\|}+\kappa\right| v_{T i}}-\frac{1}{\omega+\Omega_{i}}\right], \\
& \varepsilon_{12}^{(i)}=-\varepsilon_{21}^{(i)}=\frac{\omega_{p i}^{2}}{2 \omega}\left[\frac{\sqrt{\pi} W\left(\varsigma_{i}\right)}{\left|k_{\|}+\kappa\right| v_{T i}}-\frac{\mathrm{i}}{\omega+\Omega_{i}}\right], \\
& \varepsilon_{13}^{(i)}=\varepsilon_{23}^{(i)}=\frac{\omega_{p i}^{2}}{2 \omega^{2}} \frac{k_{r}+\mathrm{i} k_{b}}{k_{\|}+\kappa}\left[1+\mathrm{i} \sqrt{\pi} \varsigma_{i} W\left(\varsigma_{i}\right)\right], \\
& \varepsilon_{31}^{(i)}=\varepsilon_{32}^{(i)}=\frac{\omega_{p i}^{2}}{2 \omega^{2}} \frac{k_{r}-\mathrm{i} k_{b}}{k_{\|}+\kappa}\left[1+\mathrm{i} \sqrt{\pi} \varsigma_{i} W\left(\varsigma_{i}\right)\right], \\
& \varepsilon_{33}^{(i)}=-\frac{\Omega_{p i}^{2}}{\omega^{2}} .
\end{aligned}
$$

Here the ion argument of $W\left(\varsigma_{i}\right)$ - function, Eq. (18), is

$$
\varsigma_{i}=\frac{\omega-\Omega_{i}}{\left|k_{\|}+\kappa\right| v_{T i}},
$$

where the thermal velocity of resonant ions is defined by their temperature $T_{0 i}: v_{T i}=\sqrt{2 T_{0 i} / M_{i}}$.

To estimate the ICR absorption of fast waves on the fundamental cyclotron frequency $(\ell=1$, so that $\omega \sim \Omega_{i}$ ), we can use the dispersion equations (14) and/or (16), where the transverse and gyrotropic dielectric tensor components (after summation over ions and electrons) have the following form:

$$
\begin{aligned}
& \varepsilon_{\perp}=\varepsilon_{11}=\varepsilon_{22}= 1-\frac{\omega_{p i}^{2}}{2 \omega\left(\omega+\Omega_{i}\right)}+\frac{\mathrm{i} \sqrt{\pi} \omega_{p i}^{2} W\left(\varsigma_{i}\right)}{2 \omega\left(k_{\|}+\kappa\right) v_{T i}} \simeq \\
& \simeq-\frac{c^{2}}{4 v_{A}^{2}}+\frac{\mathrm{i} \sqrt{\pi} \omega_{p i}^{2} W\left(\varsigma_{i}\right)}{2 \omega\left(k_{\|}+\kappa\right) v_{T i}}, \\
& g=-i \varepsilon_{12}=i \varepsilon_{21}=-\frac{\omega_{p i}^{2}\left(2 \omega+\Omega_{i}\right)}{2 \omega \Omega_{i}\left(\omega+\Omega_{i}\right)}+\frac{\mathrm{i} \sqrt{\pi} \omega_{p i}^{2} W\left(\varsigma_{i}\right)}{2 \omega\left(k_{\|}+\kappa\right) v_{T i}} \simeq \\
& \simeq-\frac{c^{2}}{4 v_{A}^{2}}+\frac{\mathrm{i} \sqrt{\pi} \omega_{p i}^{2} W\left(\varsigma_{i}\right)}{2 \omega\left(k_{\|}+\kappa\right) v_{T i}} .
\end{aligned}
$$

As one can see these dielectric characteristics under the given (real) wave frequency $\omega$ and eigenmode numbers $m$ and $n$ have the equal anti-hermitic parts $\varepsilon_{a}$ :

$$
\varepsilon_{a}=\operatorname{Im} \varepsilon_{11}=\operatorname{Im} \varepsilon_{22}=\operatorname{Im} g=\frac{\sqrt{\pi} \omega_{p i}^{2} \exp \left(-\varsigma_{i}^{2}\right)}{2 \omega\left(k_{\|}+\kappa\right) v_{T i}},
$$

responsible [1] for the ICR absorption of both the slow and fast waves.

Assuming that $\left|\varepsilon_{\perp}\right| \simeq|g|>>N_{\|}^{2}, N_{\|} N_{0}, N_{1} N_{2}$ for fast waves with $\omega \sim \Omega_{i}$, the dispersion equation (16) can be reduced to

$$
N_{F W}^{2} \equiv N_{r}^{2}+N_{\|}^{2}+N_{b}^{2} \simeq \frac{\varepsilon_{\perp}^{2}-g^{2}}{\varepsilon_{\perp}} .
$$

Here $N=k c / \omega$ is the refractive index of fast waves (FW), $k \equiv \sqrt{k_{r}^{2}+k_{\|}^{2}+k_{b}^{2}}=\sqrt{k_{r}^{2}+n^{2} / R_{0}^{2}+m^{2} / r^{2}} \quad$ is the value of their wave vector. The dispersion characteristics of fast waves (the real and imaginary parts of the radial refractive index under the given $\omega \sim \Omega_{i}, m$ and $n$ at the considered by $r$ magnetic surface):

$$
N_{r}=\operatorname{Re} N_{r}+\mathrm{i} \operatorname{Im} N_{r}=\operatorname{Re} N_{r}(1+\mathrm{i} v)
$$

can be estimated from Eq. (26) using the smallness of ICR damping rate:

$$
v \equiv \frac{\operatorname{Im} N_{r}}{\operatorname{Re} N_{r}} \equiv \frac{\operatorname{Im} k_{r}}{\operatorname{Re} k_{r}}<<1 .
$$

As a result, the spectrum of fast waves in the ICR frequency range is defined by the expressions for these waves in the case of a uniform magnetic field:

$$
\operatorname{Re} N_{r F W}^{2}+N_{\|}^{2}+N_{b}^{2} \approx \frac{c^{2}}{v_{A}^{2}} .
$$

As we see, the radial refractive index of fast waves has a finite value for $\omega \sim \Omega_{i}$, whereas $\left|\varepsilon_{\perp}\right| \simeq|g| \rightarrow \infty$ in this frequency range. Since the transverse electric field component of fast waves and resonant ions rotate relatively $\mathbf{H}_{0}$-field lines in the opposite directions, the damping rate of fast waves should be small and can be estimated by the expression

$$
v_{F W} \approx \frac{\left|k_{\|}+\kappa\right| v_{T i} \exp \left(-\varsigma_{i}^{2}\right)}{4 \sqrt{\pi} \Omega_{i}\left|W\left(\varsigma_{i}\right)\right|^{2}} .
$$

Neglecting the $\kappa$-corrections to $k_{\|}$in Eq. (30), we obtain the well-known result for ICR fast wave damping rate in Ref. [7]. However, these $\kappa$-corrections are not important only for perturbations with $k_{\|}>h_{\theta} / r$, i.e., in the case, when the pitch of the $\mathbf{H}_{0}$-line screw is greater than the wavelength along $\mathbf{H}_{0}$. If $k_{\|} \sim h_{\theta} / r$, the $\kappa$-corrections should be accounted in the currentcarrying plasmas, including the tokamaks, or other devices, where the magnetic field is helical. The main difference between our results and the well-known ones is that fast waves propagating strictly across the uniform magnetic field $\left(k_{z}=0\right)$ are not absorbed by plasma ions, whereas if magnetic field is helical, the absorption of these waves with $k_{\|}=0$ (under $\omega \sim \Omega_{i}$ ) can be nonzero due to corrections connected with the magnetic shear and curvature of the magnetic field lines in the waveparticle resonance condition (20). In this case, the damping rate of fast waves propagating along the normal $\mathbf{n}$ to the magnetic surface in the current-carrying plasma is estimated by the formula

$$
\left.v_{F W}\right|_{k_{\|}=0} \approx\left|\frac{1}{R_{0} q}\left(1-\frac{\rho}{4 q} \frac{d q}{d \rho}\right)\right| \frac{v_{T i} \exp \left(-\varsigma_{i}^{2}\right)}{4 \sqrt{\pi} \Omega_{i}\left|W\left(\varsigma_{i}\right)\right|^{2}} .
$$

This feature of the ICR absorption of fast waves should be accounted analyzing their stability, excitation and dissipation in cylindrical and toroidal plasmas near the so-called rational magnetic surfaces, where the longitudinal wave number changes sign, i.e., $k_{\|}(r)=0$.

\section{CONCLUSIONS}

In conclusion, let us summarized the main results of our paper related to the penetration of fast waves with fixed longitudinal and poloidal mode numbers ( $n$ and $m$ ) in the frequency range below the electron cyclotron frequency $\left(\omega<\left|\Omega_{e}\right|\right)$ at the considered by $r$ magnetic surface in the cylindrical current-carrying plasma with one and/or two ion species. 
It is shown that the dielectric characteristics of electromagnetic waves in the current-carrying plasmas depend on the structure of the steady-state magnetic field configuration and plasma particle distribution functions. Spectra of fast and slow waves in a currentcarrying plasma are determined (as in a straight uniform magnetic field case) by the contribution of bulk particles to the dielectric tensor components $\varepsilon_{j k}$. However, the $\varepsilon_{j k^{-}}$ components in the current-carrying plasma (where $\mathbf{H}_{0}$ is helical), Eqs. (9), (22), (24), differ from ones for plasmas confined in the uniform magnetic field.

It is easily verify that, neglecting the poloidal magnetic field $h_{\theta} \rightarrow 0$, i.e., if $\kappa_{1}=\kappa_{2}=\kappa_{0}=\kappa \rightarrow 0$, the dispersion characteristics $\left(\operatorname{Re} N_{r}\right.$ and $\left.\operatorname{Im} N_{r}\right)$ of fast waves in the cylindrical current-carrying plasmas can be reduced to the well-known results for magnetized plasmas in a uniform magnetic field.

As in the case of a uniform magnetic field the cold plasma approximation for $\varepsilon_{j k}$ becomes incorrect nearby the Alfvén, cyclotron and hybrid resonances, where the wave phase velocity can be comparable with thermal velocities of ions and/or electrons. It means that kinetic theory should be used analyzing the wave penetration, wave excitation and wave dissipation in the currentcarrying plasmas in the range of resonant frequencies and resonant surfaces.

\section{REFERENCES}

1. A.I. Akhiezer, I.A. Akhiezer, R.V. Polovin, A.G. Sitenko, K.N. Stepanov. Plasma Electrodynamics, Pergamon. Oxford, 1975.

2. D.L. Grekov, T.D. Kaladze, K.N. Stepanov. Highfrequency heating of a finite pressure plasma by MHD waves // Sov. J. Plasma Phys. 1980, v. 6, p. 319-328. 3. D.W. Ross, G.L. Chen, S.M. Mahajan. Kinetic description of Alfvén wave heating // Phys. Fluids, 1982, v. 25, p. 652-667.

4. J. Vaclavik, K. Appert. Theory of plasma heating by low frequency waves: Magnetic pumping and Alfvén resonance heating // Nucl. Fusion. 1991, v. 31, p. 1945-1954.
5. N.N. Gorelenkov, C.Z. Cheng. Alfven cyclotron instability and ion cyclotron emission // Nucl. Fusion. 1995, v. 35, p. 1743-1752.

6. V.S. Belikov, Ya.I. Kolesnichenko, A.B. Mikhailovskii, V.A. Yavorskii. Thermonuclear Alfvenic instabilities in a reactor-tokamak // Sov. J. Plasma Phys. 1977, v. 3, p.146-155.

7. A.V. Longinov, K.N. Stepanov. HF plasma heating in tokamaks in the regions of ions cyclotron frequencies / Review. Institute of Applied Physics, Gorky, USSR, 1983, p. 105-210 (in Russian).

8. S.V. Kasilov, A.I. Piatak, K.N. Stepanov. Cyclotron resonance for fast magnetosonic waves in inhomogeneous magnetic field s of toroidal confinement devices // Nucl. Fusion. 1990, v. 30, p. 2467-2495.

9. M. Brambilla. Numerical simulation of ion cyclotron waves in tokamak plasmas // Plasma Phys. Control. Fusion. 1999, v. 41, p. 1-34.

10. M.K. Lilley, S.E. Sharapov. Compressional Alfvén and ion-ion hybrid modes in the deuterium-tritium plasma of a spherical tokamak power plant // Phys. Plasmas. 2007, v. 14, p. 082501-10

11. F.M. Nekrasov. Short-wave oscillations of a magnetized plasma with a nonuniform current // Sov. J. Plasma Phys. 1985, v. 11, p. 112-120.

12. A.G. Elfimov, C.A. de Azevedo, A.S. de Assis, N.I. Grishanov, F.M. Nekrasov, I.F. Potapenko, V.S. Tsypin. Alfvén wave heating and current drive analysis in magnetized plasma structures // Braz. J. Phys. 1995, v. 25 , p. $225-240$

13. N.I. Grishanov, A.G. Elfimov, C.A. de Azevedo, and A.S. de Assis. Short wavelength oscillations of a magnetized current-carrying plasma // Braz. J. Phys. 1996, v. 26, p. 731-741.

14. N.I. Grishanov, N.A. Azarenkov. About the cyclotron resonance conditions in magnetized currentcarrying plasmas // Phys. Plasmas. 2019, v. 26, p. 122501-9.

Article received 15.10 .2020

\section{ИОННО-ЦИКЛОТРОННОЕ ПОГЛОЩЕНИЕ БЫСТРЫХ ВОЛН В ЦИЛИНДРИЧЕСКОЙ ПЛАЗМЕ С ТОКОМ}

Н.И. Гришанов, Н.А. Азаренков

Проанализировано влияние продольного стационарного тока на поглощение и радиальную структуру быстрых волн в цилиндрической токонесушей плазме. При получении дисперсионного уравнения быстрых волн использован диэлектрический тензор, учитывающий радиальную структуру тока и геометрию удерживающего винтового магнитного поля через коэффициент запаса устойчивости плазмы. Показано, что дисперсионные характеристики быстрых волн в неравновесной плазме с током отличаются от дисперсионных характеристик равновесного плазменного шнура в однородном магнитном поле вблизи точек отсечки и резонансов из-за учета вращательного преобразования силовых линий винтового магнитного поля, включая шир-эффекты.

\section{ІОННО-ЦИКЛОТРОННЕ ПОГЛИНАННЯ ШВИДКИХ ХВИЛЬ У ЦИЛІНДРИЧНІЙ ПЛАЗМІ ЗІ СТРУМОМ}

М.I. Гришанов, М.О. Азарєнков

Проаналізовано вплив стаціонарного струму на поглинання і радіальну структуру швидких хвиль у циліндричній струмонесучій плазмі. При отриманні дисперсійного рівняння швидких хвиль використано діелектричний тензор, який враховує радіальну структуру струму і геометрію утримуючого гвинтового магнітного поля через коефіцієнт запасу стійкості плазми. Показано, що дисперсійні характеристики швидких хвиль у нерівноважній плазмі зі струмом відрізняються від дисперсійних характеристик рівноважного плазмового шнура в однорідному магнітному полі поблизу точок відсічення і резонансів через облік обертального перетворення силових ліній гвинтового магнітного поля, включаючи шир-ефекти. 